\title{
First- and Second-Moment Constrained Gaussian Channels
}

\author{
Shuai Ma*† and Michèle Wigger* \\ ${ }^{*}$ LTCI, Telecom Paris, IP Paris, 91120 Palaiseau, France \\ ${ }^{\dagger}$ National Mobile Communications Research Laboratory, Southeast University, Nanjing 210096, China \\ ${ }^{\ddagger}$ School of Information and Control Engineering, China University of Mining and Technology, Xuzhou 221116, \\ China \\ mashuai001@cumt.edu.cn, michele.wigger@telecom-paris.fr
}

\begin{abstract}
This paper studies the channel capacity of intensitymodulation direct-detection (IM/DD) visible light communication (VLC) systems under both optical and electrical power constraints. Specifically, it derives the asymptotic capacities in the high and low signal-to-noise ratio (SNR) regimes under peak, first-moment, and second-moment constraints. The results show that first- and second-moment constraints are never simultaneously active in the asymptotic low-SNR regime, and only in few cases in the asymptotic high-SNR regime. Moreover, the secondmoment constraint is more stringent in the asymptotic low-SNR regime than in the high-SNR regime.
\end{abstract}

\section{INTRODUCTION}

The ever-increasing number of wireless devices and highspeed communication requirements cause a spectrum scarcity of conventional radio-frequencies (RF). A promising solution is visible light communication (VLC) with its abundant unlicensed spectrum [1], [2]. In particular, when utilizing the simple and practical intensity modulation-direct detection (IM/DD) technology, transmitters directly modulate information onto the real, non-negative optical intensity of the VLC signals (in contrast to RF signals which modulate the complex field) and receivers apply photodetectors to measure incoming optical intensities. For eye safety reasons and hardware limitations, both the maximum and average optical intensities of VLC transmit signals typically have to be restricted. Since these apply directly to the intensities, they impose both peak and first-moment constraints on the transmit signals. Additional second-moment constraints are imposed by limitations of the electronic circuits that control the transmit signal, such as the boundedness of the linear amplification regime and electric power consumption [3]-[8].

A close-form expression for the capacity of such IM/DD systems is still unknown, even when some of the first or second-moment constraints are relaxed. However, bounds and asymptotic results in the high- and low signal-to-noise ratio (SNR) regimes are known under certain relaxations. For example, various upper and lower bounds on the capacity, as well as its exact high- and low-SNR asymptotics, have been derived under only a first-moment constraint without a second-moment constraint $[9]-[15]$.

In this work, we derive the exact expressions for the asymptotic high- and low-SNR capacities under peak, firstmoment, and second-moment constraints. Our results show that in the asymptotic low-SNR regime, only one of the two moment constraints is stringent. Specifically, the secondmoment constraint is active if the peak-constraint $A$ times the first-moment-constraint $\alpha_{1} A$ exceeds this second-moment constraint $\alpha_{2} A^{2}$, and otherwise the first-moment constraint is active. This can be seen as a consequence of the optimality of on-off keying in the asymptotic low-SNR regime. Our results further show that for most constraint-parameters $\left(\alpha_{1}, \alpha_{2}\right)$ also in the high-SNR regime, only one of the moment-constraints is active. Interestingly, the second-moment constraint is inactive over a larger region of $\left(\alpha_{1}, \alpha_{2}\right)$-pairs in the high-SNR regime than in the low-SNR regime, and the first-moment constraint over a smaller region. An additional second-moment constraint is thus more restrictive in the low-SNR regime than in the high-SNR regime. In the asymptotic high-SNR regime, we further observe a small region of $\left(\alpha_{1}, \alpha_{2}\right)$-pairs where both moment-constraints are simultaneously active and limit the asymptotic capacity.

\section{Channel Model}

Consider a typical VLC communication link, where the transmitter is equipped with a single LED or laser and the receiver with a single photodetector. The photodetector measures the incoming light intensity, which can be modeled as

$$
Y=x+Z,
$$

where $x$ denotes the input signal produced by the transmitter's LED or laser, and $Z$ is standard additive white Gaussian noise independent of $x$. Note that, in contrast to the input $x$, the output $Y$ can be negative.

Inputs $x$ are subject to both a peak and an average optical power (average-intensity) constraints:

$$
\begin{aligned}
X & \in[0, \AA], \\
\mathrm{E}[X] & \leq \alpha_{1} A,
\end{aligned}
$$

for some fixed parameters $A>0$ and $\alpha_{1} \in(0,1)$. These constraints come from (eye- and skin-) safety reasons, and from limitations (caused by non-linearities) on the optical operating regimes of LEDs and lasers.

Due to battery limitations on the attached RF circuit and power amplifier limitations, the second moment of the transmit 
signal also has to be restricted:

$$
\mathrm{E}\left[X^{2}\right] \leq \alpha_{2} \mathrm{~A}^{2}
$$

We denote the capacity of the channel (1) with allowed peak power $A$, maximum average power $\alpha_{1} A$, and maximum second moment $\alpha_{2} A^{2}$ by $\mathrm{C}\left(\alpha_{1}, \alpha_{2}, A\right)$. It is given by [16]

$$
\mathrm{C}\left(\alpha_{1}, \alpha_{2}, \mathrm{~A}\right)=\sup _{P_{X}} \mathrm{I}(X ; Y)
$$

where the supremum is over input laws $P_{X}$ satisfying (2)-(4).

Notice that, for any random variable $X \in[0, A]$, we have $\mathrm{E}\left[X^{2}\right] \leq \mathrm{E}[X] A$ and of course $\mathrm{E}[X] \leq \sqrt{\mathrm{E}\left[X^{2}\right]}$. Therefore, whenever $\alpha_{1}<\alpha_{2}$, the second moment constraint (4) is inactive in view of the first moment-constraint (3), and whenever $\sqrt{\alpha_{2}}<\alpha_{2}$, the first moment constraint (3) is inactive in view of the second moment-constraint (4). Moreover, for any $\alpha_{1} \geq 1 / 2$, the first-moment constraint (3) is not active, and for $\alpha_{2} \geq 1 / 2$, the second-moment constraint (4). In fact, by the symmetry of the Gaussian density, for any input $X$, we have $\mathrm{I}(X ; Y)=\mathrm{I}\left(X^{\prime} ; Y\right)$ for the derived input $X^{\prime}=\mathrm{A}-X$, which has smaller first and second moments than $X$ if $\mathrm{E}[X] \geq 1 / 2 \mathrm{~A}$ :

$$
\mathrm{E}\left[X^{\prime}\right]=A-\mathrm{E}[X] \leq 1 / 2 A \leq \mathrm{E}[X],
$$

and

$$
\mathrm{E}\left[X^{\prime 2}\right]=A^{2}-2 \mathrm{E}[X] A+\mathrm{E}\left[X^{2}\right] \leq \mathrm{E}\left[X^{2}\right] .
$$

We can thus limit the optimization in (5) to random variables $X$ with first moments not exceeding $1 / 2 A$ and, by $\mathrm{E}\left[X^{2}\right] \leq$ $\mathrm{E}[X] A$, with second moments not exceeding $1 / 2 A^{2}$.

As a consequence:

$\mathrm{C}\left(\alpha_{1}, \alpha_{2}, \mathrm{~A}\right)=\mathrm{C}\left(1, \alpha_{2}, \mathrm{~A}\right), \quad \forall \alpha_{1} \geq \max \left\{\sqrt{\alpha_{2}}, 1 / 2\right\}$,

and

$$
\mathrm{C}\left(\alpha_{1}, \alpha_{2}, \mathrm{~A}\right)=\mathrm{C}\left(\alpha_{1}, 1, \mathrm{~A}\right), \quad \forall \alpha_{2} \geq \max \left\{\alpha_{1}, 1 / 2\right\} .
$$

In the remainder of the paper, we present bounds on the capacities, and establish the exact asymptotic results in the high and low SNR regimes, respectively.

The following functions will be used throughout the paper. For $i=0,1,2,3,4$, define:

$$
\zeta_{i}\left(\lambda_{1}, \lambda_{2}\right):=\int_{0}^{1} y^{i} e^{-\lambda_{1} y-\lambda_{2} y^{2}} \mathrm{~d} y .
$$

\section{THE ASYMPTOTIC HIGH-SNR CAPACITY}

Consider first the asymptotic high-SNR regime, where $\alpha_{1}, \alpha_{2}$ are fixed and $\mathrm{A}$ grows without bound.

Theorem 1: Depending on the parameters $\alpha_{1}, \alpha_{2}>0$, the asymptotic high-SNR capacity satisfies one of the following limiting behaviours.

1) If $\alpha_{1} \geq \frac{1}{2}$ and $\alpha_{2} \geq \frac{1}{3}$, then both the first- and secondmoment constraints are inactive and

$$
\varlimsup_{A \rightarrow \infty}\left(\mathrm{C}\left(\alpha_{1}, \alpha_{2}, A\right)-\log \frac{A}{\sqrt{2 \pi e \sigma^{2}}}\right)=0 .
$$

2) If $0<\alpha_{1}</ 2$ is such that the unique solution $\lambda_{1}^{*}$ to the equation (in $\lambda_{1}$ )

$$
\frac{1}{\lambda_{1}}-\frac{e^{-\lambda_{1}}}{1-e^{-\lambda_{1}}}=\alpha_{1}
$$

satisfies

$$
\frac{2}{\left(\lambda_{1}^{*}\right)^{2}}-\frac{e^{-\lambda_{1}^{*}}\left(1+\frac{2}{\lambda_{1}^{*}}\right)}{1-e^{-\lambda_{1}^{*}}}<\alpha_{2},
$$

then only the first moment constraint is active and

$$
\begin{array}{r}
\varlimsup_{A \rightarrow \infty}\left(\mathrm{C}\left(\alpha_{1}, \alpha_{2}, A\right)-\log \frac{A}{\sqrt{2 \pi e \sigma^{2}}}\right) \\
=\log \zeta_{0}\left(\lambda_{1}^{*}, 0\right)+\lambda_{1}^{*} \alpha_{1} .
\end{array}
$$

3) If $0<\alpha_{2}</ 3$ is such that the unique solution $\lambda_{2}^{*}$ to the equation (in $\lambda_{2}$ )

$$
2 \sqrt{\pi \lambda_{2}}\left(\left(2 \lambda_{2}\right)^{-1}-\alpha_{2}\right)\left[\frac{1}{2}-\mathcal{Q}\left(\sqrt{2 \lambda_{2}}\right)\right]=e^{-\lambda_{2}}
$$

satisfies

$2 \sqrt{\pi \lambda_{2}^{*}} \alpha_{1}\left[\frac{1}{2}-\mathcal{Q}\left(\sqrt{2 \lambda_{2}^{*}}\right)\right]>1-e^{-\lambda_{2}^{*}}$

then only the second moment constraint is active and

$$
\begin{array}{r}
\varlimsup_{A \rightarrow \infty}\left(\mathrm{C}\left(\alpha_{1}, \alpha_{2}, A\right)-\log \frac{A}{\sqrt{2 \pi e \sigma^{2}}}\right) \\
=\log \zeta_{0}\left(0, \lambda_{2}^{*}\right)+\lambda_{2}^{*} \alpha_{2} .
\end{array}
$$

4) Else, both moment constraints are active and

$$
\begin{aligned}
& \varlimsup_{A \rightarrow \infty}(\mathrm{C}\left.\left(\alpha_{1}, \alpha_{2}, A\right)-\log \frac{A}{\sqrt{2 \pi e \sigma^{2}}}\right) \\
&=\log \zeta_{0}\left(\lambda_{1}^{*}, \lambda_{2}^{*}\right)+\lambda_{1}^{*} \alpha_{1}+\lambda_{2}^{*} \alpha_{2},
\end{aligned}
$$

for $\lambda_{1}^{*}, \lambda_{2}^{*}>0$ the unique solution to the equations

$$
\begin{aligned}
\sqrt{\pi \lambda_{2}} e^{\frac{\lambda_{1}^{2}}{4 \lambda_{2}}}\left(2 \alpha_{1}+\frac{\lambda_{1}}{\lambda_{2}}\right) & {\left[\mathcal{Q}\left(\frac{\lambda_{1}}{\sqrt{2 \lambda_{2}}}\right)-\mathcal{Q}\left(\frac{\lambda_{1}+2 \lambda_{2}}{\sqrt{2 \lambda_{2}}}\right)\right] } \\
& =1-e^{-\left(\lambda_{1}+\lambda_{2}\right)}
\end{aligned}
$$

and

$$
\begin{gathered}
\sqrt{\frac{\pi}{\lambda_{2}}} e^{\frac{\lambda_{1}^{2}}{4 \lambda_{2}}}\left(\alpha_{2}-\frac{\lambda_{2}-\lambda_{1}^{2}}{2 \lambda_{2}^{2}}\right)\left[\mathcal{Q}\left(\frac{\lambda_{1}}{\sqrt{2 \lambda_{2}}}\right)-\mathcal{Q}\left(\frac{\lambda_{1}+2 \lambda_{2}}{\sqrt{2 \lambda_{2}}}\right)\right] \\
=\frac{1}{2 \lambda_{2}} e^{-\left(\lambda_{1}+\lambda_{2}\right)}\left(\frac{\lambda_{1}}{\lambda_{2}}-1\right)-\frac{\lambda_{1}}{2 \lambda_{2}^{2}}
\end{gathered}
$$

\section{THE ASYMPTOTIC LOW-SNR CAPACITY}

Consider now the asymptotic low-SNR regime, where $\alpha_{1}, \alpha_{2}$ are again kept fixed and $\mathrm{A} \rightarrow 0$.

Proposition 2: Given parameters $\alpha_{1}, \alpha_{2}>0$,

$$
\lim _{A \downarrow 0} \frac{\mathrm{C}\left(\alpha_{1}, \alpha_{2}, A\right)}{A^{2}}=\max _{\substack{\mathrm{E}[T] \leq \alpha_{1} \\ T \in[0,1]: \frac{1}{2} \mathrm{E}\left[T^{2}\right] \leq \alpha_{2}}} \operatorname{Var}[T] .
$$

Proof: The achievability follows directly from Prelov's and Verdú's classical result on the mutual information of peak- 
constrained channels [17, Corollary 2]. The converse follows by the well-known Gaussian max-entropy bound:

$$
\mathrm{C}\left(\alpha_{1}, \alpha_{2}, A\right) \leq \max \frac{1}{2} \log \left(1+\frac{\operatorname{Var}[X]}{\sigma^{2}}\right),
$$

where the maximization is over random variables $X \in[0,1]$ satisfying (2)-(4). Defining $T:=X / A$ and using that $\lim _{t \downarrow 0} \frac{\log (1+b t)}{t}=b$, for any constant $b>0$, establishes the desired asymptotic converse bound.

Lemma 3: The maximization in Proposition 2 is attained by a binary random variable $T \in\{0, A\}$ :

$$
\max _{\substack{T \in[0,1]: \\ \mathrm{E}[T] \leq \alpha_{1} \\ \mathrm{E}\left[T^{2}\right] \leq \alpha_{2}}} \operatorname{Var}(T)=\max _{\substack{T \in\{0, \mathrm{~A}\}: \\ \mathrm{E}[T] \leq \alpha_{1} \\ \mathrm{E}\left[T^{2}\right] \leq \alpha_{2}}} \operatorname{Var}(T)
$$

Proof: Fix $T$ satisfying the conditions in the minimization and construct a new random variable $T^{\prime} \in\{0, \mathrm{~A}\}$ with $p_{\mathrm{A}}:=$ $\operatorname{Pr}\left[T^{\prime}=A\right]=\frac{\mathrm{E}\left[T^{2}\right]}{\AA^{2}}$ and $\operatorname{Pr}\left[T^{\prime}=0\right]=1-p_{\AA}$. Notice that $\mathrm{E}\left[\left(T^{\prime}\right)^{2}\right]=p_{\mathrm{A}} A^{2}=\mathrm{E}\left[T^{2}\right]$ and

$$
\mathrm{E}\left[T^{\prime}\right]=p_{\text {A }} \mathrm{A}=\frac{\mathrm{E}\left[T^{2}\right]}{\mathrm{A}} \leq \frac{\mathrm{E}[T] \cdot \mathrm{A}}{\mathrm{A}}=\mathrm{E}[T] .
$$

The new random variable $T^{\prime}$ thus also satisfies the conditions in the maximization, and moreover it has larger objective function (variance) than $T$ because $\operatorname{Var}\left[T^{\prime}\right]=\mathrm{E}\left[\left(T^{\prime}\right)^{2}\right]-$ $\left(\mathrm{E}\left[T^{\prime}\right]\right)^{2} \geq \mathrm{E}\left[(T)^{2}\right]-(\mathrm{E}[T])^{2}=\operatorname{Var}[T]$.

Combining Proposition 2 with Lemma 3 establishes the desired low-SNR asymptotics.

Theorem 4: For any parameters $\alpha_{1}, \alpha_{2}>0$ :

$$
\lim _{\mathrm{A} \downarrow 0} \frac{\mathrm{C}\left(\alpha_{1}, \alpha_{2}, \mathrm{~A}\right)}{\mathrm{A}^{2}}=p^{*}\left(1-p^{*}\right)
$$

where $p^{*}:=\min \left\{\alpha_{1}, \alpha_{2}, 1 / 2\right\}$.

Proof: By Lemma 3 .

$$
\max _{\substack{T \in[0,1]: \\ E[T] \leq \alpha_{1} \\ \mathrm{E}\left[T^{2}\right] \leq \alpha_{2}}} \operatorname{Var}(T)=\max _{\substack{p_{\mathcal{A}} \in[0,1]: \\ p_{\boldsymbol{A}} \leq \alpha_{1} \\ p_{\text {A }} \leq \alpha_{2}}} p_{\text {A }}\left(1-p_{\text {A }}\right) .
$$

Since the function $t \mapsto t(1-t)$ is continuous and monotonically increasing over $[0,1 / 2]$ but monotonically decreasing over $[1 / 2,1]$, the maximum value is obtained for $p_{\mathrm{A}}=$ $\min \left\{\alpha_{1}, \alpha_{2}, 1 / 2\right\}$. Plugging this into Proposition 2 establishes the desired result.

\section{Discussion of Asymptotic Results}

Figure 1 illustrates the regions of $\left(\alpha_{1}, \alpha_{2}\right)$-pairs where both the first and the second-moment constraints, i.e., (3) and (4), are active. At any SNR values, the first-moment constraint (3) is not active on the right of the the blue dash-dotted line, see (8), and the second-moment constraint (3) is not active above the red dash-dotted line, see (9).

In the asymptotic low-SNR regime, only one of the two constraints is active, unless $\alpha_{1}=\alpha_{2}<1 / 2$ in which case both constraints are active, or $\alpha_{1}, \alpha_{2} \geq 1 / 2$ in which case no constraint is active. Otherwise, the first-moment constraint is active when $\alpha_{1}<\min \left\{\alpha_{2}, 1 / 2\right\}$, i.e., above the dashdotted

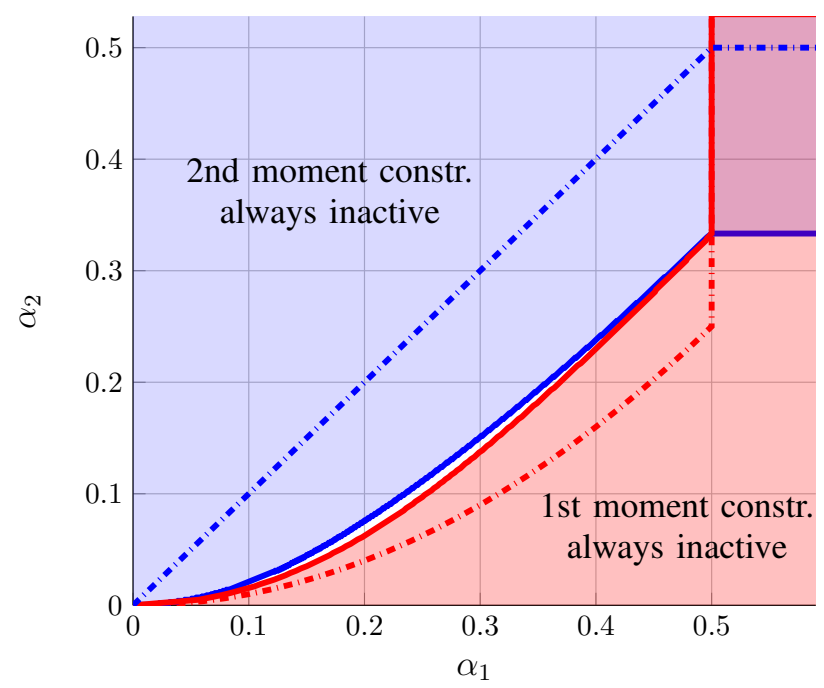

Fig. 1: The figure illustrates the regions where the two moment constraints (3) and (4) limit the (asymptotic) capacity.

blue line, and the second-moment constraint is active when $\alpha_{2}<\min \left\{\alpha_{1}, 1 / 2\right\}$, i.e., below the dashdotted blue line.

This contrasts the high-SNR regime where both constraints are inactive for $\alpha_{1} \geq 1 / 2$ and $\alpha_{2} \geq 1 / 3$. Generally, the firstmoment constraint (3) is inactive for all $\left(\alpha_{1}, \alpha_{2}\right)$-pairs on the right of the red solid line shown in Figure 1 The secondmoment constraint (4) is inactive for all pairs lying above the blue solid line. Both the first and second-moment constraints are thus simultaneously active only in the small white region that lies in between the solid blue and red lines.

Overall, it can be noted that the second-moment constraint is more stringent in the low-SNR regime than in the highSNR regime, where it is inactive for more $\left(\alpha_{1}, \alpha_{2}\right)$-pairs. Surprisingly, we observe that in the asymptotic regimes both constraints are simultaneously active in very few cases.

\section{Proof of Theorem 1}

\section{A. Lower Bound}

We first lower-bound the capacity with some simple entropy-manipulations and by using the entropy-maximizing input-density $f_{X}^{*}(x)$ over $[0, A]$. Under constraints (2)-(4), $f_{X}^{*}(x)$ has the form:

$$
f_{X}^{*}(x)=\left(A \zeta_{0}\left(\lambda_{1}, \lambda_{2}\right)\right)^{-1} \cdot e^{-\frac{\lambda_{1}}{A} x-\frac{\lambda_{2}}{A^{2}} x^{2}}, \quad x \in[0, A],
$$

where the parameters $\lambda_{1}, \lambda_{2}$ have to be chosen to satisfy

$$
\begin{aligned}
& \int_{0}^{\mathrm{A}} f_{X}^{*}(x) \cdot x \mathrm{~d} x \leq \alpha_{1} A, \\
& \int_{0}^{\mathrm{A}} f_{X}^{*}(x) \cdot x^{2} \mathrm{~d} x \leq \alpha_{2} A^{2} .
\end{aligned}
$$

Given the form in (21), through a simple variable substitution $y=\frac{x}{\mathrm{~A}}$, one can prove that (22) are equivalent to

$$
\begin{aligned}
& \frac{\zeta_{1}\left(\lambda_{1}, \lambda_{2}\right)}{\zeta_{0}\left(\lambda_{1}, \lambda_{2}\right)} \leq \alpha_{1}, \\
& \frac{\zeta_{2}\left(\lambda_{1}, \lambda_{2}\right)}{\zeta_{0}\left(\lambda_{1}, \lambda_{2}\right)} \leq \alpha_{2},
\end{aligned}
$$


where recall that the functions $\zeta_{i}$, for $i=0,1, \ldots, 4$, are defined in (10). Then,

$$
\begin{aligned}
\mathrm{C}( & \left.\alpha_{1}, \alpha_{2}, \mathrm{~A}\right) \\
\geq & I_{f_{X}^{*}}(X ; Y) \\
= & h_{f_{X}^{*}}(Y)-h(Z) \geq h_{f_{X}^{*}}(Y \mid Z)-h(Z) \\
= & h_{f_{X}^{*}}(X)-h(Z) \\
= & \mathrm{E}_{f_{X}^{*}}\left[-\log f_{X}^{*}(X)\right]-\frac{1}{2} \log \left(2 \pi e \sigma^{2}\right) \\
= & \log \left(\mathrm{A} \cdot \zeta_{0}\left(\lambda_{1}, \lambda_{2}\right)\right)+\frac{\lambda_{1}}{\mathrm{~A}} \mathrm{E}_{f_{X}^{*}}[X]+\frac{\lambda_{2}}{\mathrm{~A}^{2}} \mathrm{E}_{f_{X}^{*}}\left[X^{2}\right] \\
& -\frac{1}{2} \log \left(2 \pi e \sigma^{2}\right) \\
= & \log \left(\frac{\mathrm{A} \cdot \zeta_{0}\left(\lambda_{1}, \lambda_{2}\right)}{\sqrt{2 \pi e \sigma^{2}}}\right)+\lambda_{1} \alpha_{1}+\lambda_{2} \alpha_{2}
\end{aligned}
$$

where all $\left(\lambda_{1}, \lambda_{2}\right)$ satisfying (23) yield valid lower bounds.

\section{B. Upper bound}

We turn to the duality-based upper bound with the choice of output density

$$
f_{Y}(y)=\tau \cdot f_{Y}^{(1)}(y)+(1-\tau) \cdot f_{Y}^{(2)}(y),
$$

where $\tau \in(0,1)$ is a parameter that we specify later on; $f_{Y}^{(1)}(y)$ is a probability density function over the interval $\mathcal{I}:=$ $[0, A]$ of the form

$$
f_{Y}^{(1)}(y)=\frac{1}{\mathrm{~A} \cdot \zeta_{0}\left(\lambda_{1}, \lambda_{2}\right)} e^{-\frac{\lambda_{1}}{\mathrm{~A}} y-\frac{\lambda_{2}}{\mathrm{~A}} y^{2}} \cdot \mathbb{1}\{y \in \mathcal{I}\},
$$

where $\lambda_{1}, \lambda_{2} \geq 0$ are free parameters, over which we will optimize in a latter stage; and $f_{Y}^{(2)}(y)$ is a probability density function over the rest of the real line $\mathcal{I}^{c}:=\mathbb{R} \backslash \mathcal{I}$ :

$$
f_{Y}^{(2)}(y)= \begin{cases}\frac{1}{\sqrt{2 \pi \sigma^{2}}} e^{-\frac{y^{2}}{2 \sigma^{2}}} & \text { if } y<0, \\ \frac{1}{\sqrt{2 \pi \sigma^{2}}} e^{-\frac{(y-A)^{2}}{2 \sigma^{2}}} & \text { if } y>A .\end{cases}
$$

For the choice in (30), the duality-based upper bound yields

$$
\begin{aligned}
\mathrm{C} & \left(\alpha_{1}, \alpha_{2}, \mathrm{~A}\right) \\
\leq & \mathrm{E}_{f_{Y}^{*}}\left[-\log f_{Y}(Y)\right]-\frac{1}{2} \log \left(2 \pi e \sigma^{2}\right) \\
\leq & \mathrm{E}_{f_{Y}^{*}}\left[-\log \left(\tau f_{Y}^{(1)}(Y)\right) \mid Y \in \mathcal{I}\right] \cdot P_{f_{Y}^{*}}(\mathcal{I}) \\
& +\mathrm{E}_{f_{Y}^{*}}\left[-\log \left((1-\tau) f_{Y}^{(2)}(Y)\right) \mid Y \in \mathcal{I}^{c}\right] \cdot P_{f_{Y}^{*}}\left(\mathcal{I}^{c}\right) \\
& -\frac{1}{2} \log \left(2 \pi e \sigma^{2}\right) \\
= & \log \frac{\mathrm{A} \cdot \zeta_{0}\left(\lambda_{1}, \lambda_{2}\right)}{\tau} \cdot P_{f_{Y}^{*}}(\mathcal{I}) \\
& +\mathrm{E}_{f_{Y}^{*}}\left[-\log \left(\tau \cdot f_{Y}^{(2)}(Y)\right) \mid Y \in \mathcal{I}^{c}\right] \cdot P_{f_{Y}^{*}}\left(\mathcal{I}^{c}\right) \\
& +\left(\frac{\lambda_{1}}{\mathrm{~A}} \mathrm{E}_{f_{Y}^{*}}[Y \mid Y \in \mathcal{I}]+\frac{\lambda_{2}}{A^{2}} \mathrm{E}_{f_{Y}^{*}}\left[Y^{2} \mid Y \in \mathcal{I}\right]\right) \cdot P_{f_{Y}^{*}}(\mathcal{I}) \\
& -\frac{1}{2} \log \left(2 \pi e \sigma^{2}\right)
\end{aligned}
$$

Following similar steps as, e.g., in [18, Eq. (209)-(226)], we obtain the following lemmas.
Lemma 5: For the Gaussian-tail distribution defined in (32):

$$
\mathrm{E}_{f_{Y}^{*}}\left[-\log \left(f_{Y}^{(2)}(Y)\right) \mid Y \in \mathcal{I}^{c}\right] \leq \log \sqrt{2 \pi e \sigma^{2}} .
$$

Proof: We have

$$
\begin{aligned}
& \int_{-\infty}^{0} \frac{1}{\sqrt{2 \pi}} e^{-\frac{(y-x)^{2}}{2}}\left(\log \sqrt{2 \pi}+\frac{y^{2}}{2}\right) \mathrm{d} y \\
& =\log \sqrt{2 \pi} \mathcal{Q}(x)+\frac{1}{2} x^{2} \mathcal{Q}(x)+\frac{1}{2} \mathcal{Q}(x)-\frac{x}{2} \frac{1}{\sqrt{2 \pi}} e^{-\frac{x^{2}}{2}} \\
& \leq\left(\log \sqrt{2 \pi}+\frac{1}{2}\right) \mathcal{Q}(x)
\end{aligned}
$$

ans similarly

$$
\begin{aligned}
& \int_{\mathrm{A}}^{\infty} \frac{1}{\sqrt{2 \pi}} e^{-\frac{(y-x)^{2}}{2}}\left(\log \sqrt{2 \pi}+\frac{(y-A)^{2}}{2}\right) \mathrm{d} y \\
& \leq\left(\log \sqrt{2 \pi}+\frac{1}{2}\right) \mathcal{Q}(\mathrm{A}-x) .
\end{aligned}
$$

Therefore,

$$
\begin{aligned}
\mathrm{E}_{f_{Y}^{*}} & {\left[-\log \left(f_{Y}^{(2)}(Y)\right) \mid Y \in \mathcal{I}^{c}\right] \cdot P_{f_{Y}^{*}}\left(\mathcal{I}^{c}\right) } \\
& =-\int_{\mathcal{I}^{c}} f_{Y}^{*}(y) \log \left(f_{Y}^{(2)}(Y)\right) \mathrm{d} y \\
& =-\int_{\mathcal{I}^{c}} \int_{0}^{\mathrm{A}} f_{X}^{*}(x) \frac{1}{\sqrt{2 \pi}} e^{-\frac{(y-x)^{2}}{2}} \mathrm{~d} x \cdot \log \left(f_{Y}^{(2)}(Y)\right) \mathrm{d} y \\
& =-\mathrm{E}_{f_{X}^{*}}\left[\int_{\mathcal{I}^{c}} \frac{1}{\sqrt{2 \pi}} e^{-\frac{(y-X)^{2}}{2}} \log \left(f_{Y}^{(2)}(Y)\right) \mathrm{d} y\right] \\
& =\left(\log \sqrt{2 \pi} e+\frac{1}{2}\right) \mathrm{E}_{f_{X}^{*}}[\mathcal{Q}(X)+\mathcal{Q}(A-X)] .
\end{aligned}
$$

Since

$$
\begin{aligned}
P_{f_{Y}^{*}}\left(\mathcal{I}^{c}\right) & =\mathrm{E}_{f_{X}^{*}}\left[\int_{\mathcal{I}^{c}} \frac{1}{\sqrt{2 \pi}} e^{-\frac{(y-X)^{2}}{2}} \mathrm{~d} y\right] \\
& =\mathrm{E}_{f_{X}^{*}}[\mathcal{Q}(X)+\mathcal{Q}(\mathrm{A}-X)]
\end{aligned}
$$

we obtain the desired result.

Lemma 6: For the distribution in (31):

$$
\begin{aligned}
\mathrm{E}_{f_{Y}^{*}}[Y \mid Y \in \mathcal{I}] \cdot P_{f_{Y}^{*}}(\mathcal{I}) & \leq \mathrm{E}_{f_{Y}^{*}}[Y]+\frac{1}{\sqrt{2 \pi}}\left(1-e^{-\frac{\mathrm{A}^{2}}{2}}\right) \\
& =\mathrm{E}_{f_{X}^{*}}[X]+\left(1-\frac{1}{\sqrt{2 \pi}} e^{-\frac{\mathrm{A}^{2}}{2}}\right)
\end{aligned}
$$

and

$\mathrm{E}_{f_{Y}^{*}}\left[Y^{2} \mid Y \in \mathcal{I}\right] \cdot P_{f_{Y}^{*}}(\mathcal{I}) \leq \mathrm{E}_{f_{Y}^{*}}\left[Y^{2}\right]=\mathrm{E}_{f_{X}^{*}}\left[X^{2}\right]+\sigma^{2}$.

Proof: The inequality in (49) follows simply because $Y^{2} \geq 0$ with probability 1 . The inequality in (47) is proved as follows:

$$
\begin{aligned}
\mathrm{E}_{f_{Y}^{*}} & {[Y \mid Y \in \mathcal{I}] \cdot P_{f_{Y}^{*}}(\mathcal{I}) } \\
& =\int_{0}^{\mathrm{A}} f_{Y}^{*}(y) \cdot y \mathrm{~d} y \\
& =\int_{0}^{\mathrm{A}} \int_{0}^{\mathrm{A}} f_{X}^{*}(x) \frac{1}{\sqrt{2 \pi}} e^{-\frac{(y-x)^{2}}{2}} \mathrm{~d} x \cdot y \mathrm{~d} y
\end{aligned}
$$




$$
\begin{aligned}
= & \mathrm{E}_{f_{X}^{*}}\left[\int_{0}^{\mathrm{A}} \frac{1}{\sqrt{2 \pi}} e^{-\frac{(y-X)^{2}}{2}} y \mathrm{~d} y\right] \\
= & \mathrm{E}_{f_{X}^{*}}[X(1-\mathcal{Q}(X)-\mathcal{Q}(\mathrm{A}-X))] \\
& +\mathrm{E}_{f_{X}^{*}}\left[\left(\frac{1}{\sqrt{2 \pi}} e^{-\frac{X^{2}}{2}}-\frac{1}{\sqrt{2 \pi}} e^{-\frac{(A-X)^{2}}{2}}\right)\right] \\
< & \mathrm{E}_{f_{X}^{*}}[X]+\frac{1}{\sqrt{2 \pi}} \cdot\left(1-e^{-\frac{A^{2}}{2}}\right),
\end{aligned}
$$

where the last inequality holds because the $\mathcal{Q}(\cdot)$-function is positive and the exponential function monotonically increasing.

We continue with our upper bound. By plugging these lemmas into 36 and choosing

$$
\tau=\frac{\mathrm{A} \cdot \zeta_{0}\left(\lambda_{1}, \lambda_{2}\right)}{\mathrm{A} \cdot \zeta_{0}\left(\lambda_{1}, \lambda_{2}\right)+\sqrt{2 \pi e \sigma^{2}}},
$$

we obtain:

$$
\begin{aligned}
\mathrm{C}\left(\alpha_{1},\right. & \left.\alpha_{2}, \mathrm{~A}\right) \\
\leq & \log \frac{\mathrm{A} \cdot \zeta_{0}\left(\lambda_{1}, \lambda_{2}\right)}{\tau} \cdot P_{f_{Y}^{*}}(\mathcal{I})+\log \frac{\sqrt{2 \pi e \sigma^{2}}}{1-\tau} \cdot P_{f_{Y}^{*}}\left(\mathcal{I}^{c}\right) \\
& +\frac{\lambda_{1}}{\mathrm{~A}} \mathrm{E}_{f_{X}^{*}}[X]+\frac{\lambda_{2}}{\mathrm{~A}^{2}}\left(\mathrm{E}_{f_{X}^{*}}\left[X^{2}\right]+\sigma^{2}\right) \\
& +\frac{\lambda_{1}}{\mathrm{~A}}\left(1-\frac{1}{\sqrt{2 \pi}} e^{-\frac{\mathrm{A}^{2}}{2}}\right)-\frac{1}{2} \log \left(2 \pi e \sigma^{2}\right) \\
= & \log \left(\mathrm{A} \cdot \zeta_{0}\left(\lambda_{1}, \lambda_{2}\right)+\sqrt{2 \pi e \sigma^{2}}\right) \cdot P_{f_{Y}^{*}}(\mathcal{I}) \\
& +\log \left(\mathrm{A} \cdot \zeta_{0}\left(\lambda_{1}, \lambda_{2}\right)+\sqrt{2 \pi e \sigma^{2}}\right) \cdot P_{f_{Y}^{*}}\left(\mathcal{I}^{c}\right) \\
& +\frac{\lambda_{1}}{\mathrm{~A}} \mathrm{E}_{f_{X}^{*}}[X]+\frac{\lambda_{2}}{\mathrm{~A}^{2}}\left(\mathrm{E}_{f_{X}^{*}}\left[X^{2}\right]+\sigma^{2}\right) \\
& +\frac{\lambda_{1}}{\mathrm{~A}}\left(1-\frac{1}{\sqrt{2 \pi}} e^{-\frac{\mathrm{A}^{2}}{2}}\right)-\frac{1}{2} \log \left(2 \pi e \sigma^{2}\right) \\
\leq & \log \left(\mathrm{A} \cdot \zeta_{0}\left(\lambda_{1}, \lambda_{2}\right)+\sqrt{2 \pi e \sigma^{2}}\right)+\lambda_{1} \alpha_{1}+\lambda_{2} \alpha_{2}+\lambda_{2} \frac{\sigma^{2}}{\mathrm{~A}^{2}} \\
& +\frac{\lambda_{1}}{\mathrm{~A}}\left(1-\frac{1}{\sqrt{2 \pi}} e^{-\frac{\mathrm{A}^{2}}{2}}\right)-\frac{1}{2} \log \left(2 \pi e \sigma^{2}\right) \\
= & \log \left(1+\frac{\mathrm{A} \cdot \zeta_{0}\left(\lambda_{1}, \lambda_{2}\right)}{2 \pi e \sigma^{2}}\right)+\lambda_{1} \alpha_{1}+\lambda_{2} \alpha_{2} \\
& +\lambda_{2} \frac{\sigma^{2}}{\mathrm{~A}^{2}}+\frac{\lambda_{1}}{\mathrm{~A}}\left(1-\frac{1}{\sqrt{2 \pi}} e^{-\frac{\mathrm{A}^{2}}{2}}\right) .
\end{aligned}
$$

We can conclude that for any choice of $\lambda_{1}, \lambda_{2} \geq 0$ :

$$
\begin{array}{r}
\varlimsup_{A \rightarrow \infty}\left(\mathrm{C}\left(\alpha_{1}, \alpha_{2}, A\right)-\log \frac{\mathrm{A}}{\sqrt{2 \pi e \sigma^{2}}}\right) \\
\leq \log \zeta_{0}\left(\lambda_{1}, \lambda_{2}\right)+\lambda_{1} \alpha_{1}+\lambda_{2} \alpha_{2} .
\end{array}
$$

\section{Distinction of the Four Cases}

We now show that the case distinction proposed in the theorem partitions the set of all $\left(\alpha_{1}, \alpha_{2}\right)$-parameters and that the described choice of $\lambda_{1}^{*}, \lambda_{2}^{*}$-parameters exists in each subset. More specifically, we show that the proposed case distinction coincides with the case distinction that arises when minimizing the right-hand side of [60), i.e., the function

$$
\Gamma\left(\lambda_{1}, \lambda_{2}\right):=\log \zeta_{0}\left(\lambda_{1}, \lambda_{2}\right)+\lambda_{1} \alpha_{1}+\lambda_{2} \alpha_{2},
$$

over the choices $\lambda_{1}, \lambda_{2}>0$, and we show that the $\lambda_{1}^{*}, \lambda_{2}^{*}$ values given in the theorem are the minimizers of this function.

Consider the partial derivatives of this function:

$$
\frac{\partial \Gamma}{\partial \lambda_{1}}=-\frac{\zeta_{1}\left(\lambda_{1}, \lambda_{2}\right)}{\zeta_{0}\left(\lambda_{1}, \lambda_{2}\right)}+\alpha_{1}
$$

and

$$
\frac{\partial \Gamma}{\partial \lambda_{2}}=-\frac{\zeta_{2}\left(\lambda_{1}, \lambda_{2}\right)}{\zeta_{0}\left(\lambda_{1}, \lambda_{2}\right)}+\alpha_{2},
$$

as well as its Hessian matrix

$$
\begin{aligned}
& \mathbb{H} \Gamma\left(\lambda_{1}, \lambda_{2}\right):=\left(\begin{array}{cc}
\frac{\partial^{2} \Gamma\left(\lambda_{1}, \lambda_{2}\right)}{\partial \lambda_{1}^{2}} & \frac{\partial^{2} \Gamma\left(\lambda_{1}, \lambda_{2}\right)}{\partial \lambda_{1} \partial \lambda_{2}} \\
\frac{\partial^{2} \Gamma\left(\lambda_{1}, \lambda_{2}\right)}{\partial \lambda_{1} \partial \lambda_{2}} & \frac{\partial^{2} \Gamma\left(\lambda_{1}, \lambda_{2}\right)}{\partial \lambda_{2}^{2}}
\end{array}\right) \\
& =\left(\begin{array}{cc}
\zeta_{2}\left(\lambda_{1}, \lambda_{2}\right)-\zeta_{1}^{2}\left(\lambda_{1}, \lambda_{2}\right) & c\left(\lambda_{1}, \lambda_{2}\right) \\
c\left(\lambda_{1}, \lambda_{2}\right) & \zeta_{4}\left(\lambda_{1}, \lambda_{2}\right)-\zeta_{2}^{2}\left(\lambda_{1}, \lambda_{2}\right)
\end{array}\right),
\end{aligned}
$$

where

$$
c\left(\lambda_{1}, \lambda_{2}\right):=\zeta_{3}\left(\lambda_{1}, \lambda_{2}\right)-\zeta_{1}\left(\lambda_{1}, \lambda_{2}\right) \cdot \zeta_{2}\left(\lambda_{1}, \lambda_{2}\right) .
$$

Since for any pair $\left(\lambda_{1}, \lambda_{2}\right)$ the Hessian $\mathbb{H} \Gamma\left(\lambda_{1}, \lambda_{2}\right)$ is a twoby-two matrix with positive trace and determinant, all its eigenvalues are positive, and the Hessian itself is positive definite for all $\left(\lambda_{1}, \lambda_{2}\right)$. As a consequence, the function $\Gamma\left(\lambda_{1}, \lambda_{2}\right)$ is jointly strictly convex in both arguments and the minimizer $\left(\lambda_{1}^{*}, \lambda_{2}^{*}\right)$ of $\Gamma\left(\lambda_{1}, \lambda_{2}\right)$, for $\lambda_{1}, \lambda_{2} \geq 0$ is accordingly obtained as follows, depending on the values of $\alpha_{1}$ and $\alpha_{2}$ :

1) If both partial derivatives of $\Gamma$ at the origin are strictly positive, i.e.,

$$
\begin{gathered}
-\frac{\zeta_{1}(0,0)}{\zeta_{0}(0,0)}+\alpha_{1}=-\frac{1}{2}+\alpha_{1}>0, \\
-\frac{\zeta_{2}(0,0)}{\zeta_{0}(0,0)}+\alpha_{2}=-\frac{1}{3}+\alpha_{2}>0,
\end{gathered}
$$

then $\lambda_{1}^{*}=\lambda_{2}^{*}=0$.

2) If for some $\lambda_{1}^{\prime}>0$ the partial derivatives of $\Gamma$ satisfy

$$
\begin{aligned}
& -\frac{\zeta_{1}\left(\lambda_{1}^{\prime}, 0\right)}{\zeta_{0}\left(\lambda_{1}^{\prime}, 0\right)}+\alpha_{1}=0, \\
& -\frac{\zeta_{2}\left(\lambda_{1}^{\prime}, 0\right)}{\zeta_{0}\left(\lambda_{1}^{\prime}, 0\right)}+\alpha_{2}>0,
\end{aligned}
$$

then $\lambda_{1}^{*}=\lambda_{1}^{\prime}$ and $\lambda_{2}^{*}=0$.

3) If for some $\lambda_{2}^{\prime}>0$ the partial derivatives of $\Gamma$ satisfy

$$
\begin{aligned}
& -\frac{\zeta_{1}\left(0, \lambda_{2}^{\prime}\right)}{\zeta_{0}\left(0, \lambda_{2}^{\prime}\right)}+\alpha_{1}>0 \\
& -\frac{\zeta_{2}\left(0, \lambda_{2}^{\prime}\right)}{\zeta_{0}\left(0, \lambda_{2}^{\prime}\right)}+\alpha_{2}=0,
\end{aligned}
$$

then $\lambda_{1}^{*}=0$ and $\lambda_{2}^{*}=\lambda_{2}^{\prime}$.

4) If for some $\lambda_{1}^{\prime}, \lambda_{2}^{\prime}>0$ the partial derivatives of $\Gamma$ at $\left(\lambda_{1}^{\prime}, \lambda_{2}^{\prime}\right)$ are both zero, i.e.,

$$
\begin{aligned}
& -\frac{\zeta_{1}\left(\lambda_{1}^{\prime}, \lambda_{2}^{\prime}\right)}{\zeta_{0}\left(\lambda_{1}^{\prime}, \lambda_{2}^{\prime}\right)}+\alpha_{1}=0 \\
& -\frac{\zeta_{2}\left(\lambda_{1}^{\prime}, \lambda_{2}^{\prime}\right)}{\zeta_{0}\left(\lambda_{1}^{\prime}, \lambda_{2}^{\prime}\right)}+\alpha_{2}=0,
\end{aligned}
$$




$$
\text { then } \lambda_{1}^{*}=\lambda_{1}^{\prime} \text { and } \lambda_{2}^{*}=\lambda_{2}^{\prime} \text {. }
$$

Since the strictly convex function $\Gamma\left(\lambda_{1}, \lambda_{2}\right)$ has exactly one minimizing pair, combined with continuity considerations, this concludes the proof of the theorem.

\section{ACKNOWLEDGEMENT}

The authors thank Lina Mroueh for interesting discussions.

\section{REFERENCES}

[1] A. Jovicic, J. Li, and T. Richardson, "Visible light communication: opportunities, challenges and the path to market," IEEE Commun. Mag., vol. 51, no. 12, pp. 26-32, Dec. 2013.

[2] P. H. Pathak, X. Feng, P. Hu, and P. Mohapatra, "Visible light communication, networking, and sensing: a survey, potential and challenges," IEEE Commun. Surveys Tuts., vol. 17, no. 4, pp. 2047-2077, Sept. 2015.

[3] "IEEE standard for local and metropolitan area networks-part 15.7: Short-range wireless optical communication using visible light," IEEE Std 802.15.7-2011, pp. 1-309, 2011.

[4] S. Ma, H. Li, Y. He, R. Yang, S. Lu, W. Cao, and S. Li, "Capacity bounds and interference management for interference channel in visible light communication networks," IEEE Trans. Wireless Commun., vol. 18, no. 1, pp. 182-193, Jan. 2019.

[5] X. Ling, J. Wang, X. Liang, Z. Ding, and C. Zhao, "Offset and power optimization for DCO-OFDM in visible light communication systems," IEEE Trans. Signal Process., vol. 64, no. 2, pp. 349-363, Jan. 2016.

[6] X. Huang, J. Shi, J. Li, Y. Wang, and N. Chi, "A Gb/s VLC transmission using hardware preequalization circuit," IEEE Photon. Technol. Lett., vol. 27, no. 18, pp. 1915-1918, Sep. 2015.

[7] F. Che, L. Wu, B. Hussain, X. Li, and C. P. Yue, "A fully integrated IEEE 802.15.7 visible light communication transmitter with on-chip 8W 85\% efficiency boost LED driver," J. Lightw. Technol., vol. 34, no. 10 , pp. 2419-2430, 2016.

[8] S. Ma, R. Yang, H. Li, Z.-L. Dong, H. Gu, and S. Li, "Achievable rate with closed-form for SISO channel and broadcast channel in visible light communication networks," J. Lightw. Technol., vol. 35, no. 14, pp. 2778-2787, Jul. 2017.

[9] A. Lapidoth, S. M. Moser, and M. Wigger, "On the capacity of freespace optical intensity channels," IEEE Trans. Inf. Theory, vol. 55, no. 10, pp. 4449-4461, Oct. 2009.

[10] A. Chaaban, J. Morvan, and M. Alouini, "Free-space optical communications: capacity bounds, approximations, and a new sphere-packing perspective," IEEE Trans. Commun., vol. 64, no. 3, pp. 1176-1191, Mar. 2016.

[11] J. B. Wang, Q. S. Hu, J. Wang, M. Chen, and J. Y. Wang, "Tight bounds on channel capacity for dimmable visible light communications," $J$. Lightw. Technol., vol. 31, no. 23, pp. 3771-3779, Dec. 2013.

[12] T. Chan, S. Hranilovic, and F. Kschischang, "Capacity-achieving probability measure for conditionally Gaussian channels with bounded inputs," IEEE Trans. Inf. Theory, vol. 51, no. 6, pp. 2073-2088, Jun. 2005.

[13] R. Jiang, Z. Wang, Q. Wang, and L. Dai, "A tight upper bound on channel capacity for visible light communications," IEEE Commun. lett., vol. 20, no. 1, pp. 1089-7798, Jan. 2016.

[14] A. A. Farid and S. Hranilovic, "Capacity bounds for wireless optical intensity channels with Gaussian noise," IEEE Trans. Inf. Theory, vol. 56, no. 12, pp. 6066-6077, Dec. 2010.

[15] A. A. Farid and S. Hranilovic, "Channel capacity and non-uniform signaling for free-space optical intensity channels," IEEE J. Sel. Areas Commun., vol. 17, no. 9, pp. 1553-1563, Dec. 2009.

[16] T. M. Cover and J. A. Thomas, Elements of Information Theory, 2nd ed., New York:Wiley, 2006.

[17] V. V. Prelov and S. Verdú, "Second-order asymptotics of mutual information," IEEE Trans. Inf. Theory, vol. 50, no. 8, pp. 1567-1580, Aug. 2004.

[18] L. Li, S. M. Moser, L. Wang, and M. Wigger, "On the capacity of MIMO optical wireless channels," IEEE Trans. Inf. Theory, vol. 66, no. 9, pp. 5660-5682, Sep. 2020. 